\title{
3 Research Square \\ OSR2Vec: an Ontology Semantic Representation Method Based Pre-training Natural Language Model
}

\author{
Yun Liu ( $\nabla$ liuyun@njmu.edu.cn ) \\ Nanjing Medical University \\ Huiting Sun \\ Nanjing Medical University \\ Junjie Wang \\ Nanjing Medical University \\ Jie Hu \\ Nanjing Medical University \\ Wei Feng \\ Nanjing Medical University \\ Xinyi Cao \\ Nanjing Medical University
}

\section{Research Article}

Keywords:

Posted Date: March 3rd, 2022

DOI: https://doi.org/10.21203/rs.3.rs-1379598/v1

License: (c) (i) This work is licensed under a Creative Commons Attribution 4.0 International License.

Read Full License 


\section{RESEARCH}

\section{OSR2Vec: an Ontology Semantic Representation Method Based Pre-training Natural Language Model}

Huiting Sun ${ }^{1}$, Junjie Wang ${ }^{1,2}$, Jie Hu ${ }^{1,2}$, Wei Feng ${ }^{1}$, Xinyi Cao ${ }^{1}$ and Yun $\mathrm{Liu}^{1,2^{*}}$

${ }^{*}$ Correspondence:
liuyun@njmu.edu.cn
${ }^{1}$ Department of Medical
Informatics, School of Biomedical
Engineering and Informatics,
Nanjing Medical University,
Nanjing, China
Full list of author information is
available at the end of the article

\begin{abstract}
Background: Ontology, as a formal description and organization for domain-specific knowledge, is served as a significant approach of information retrieval and biological discovery in biomedical research, attracting more and more attention in computational biomedical and bioinformatics. It is quantified entities using semantic similarity based on ontology terms when using ontologies for description or annotation. More recently, machine learning approaches have enabled ontologies to describe concepts in "computable" semantics. However, ontology semantics representation is not yet well-explored by existing learning-based methods.

Results: We propose OSR2Vec, an ontology semantic representation approach that includes transforming structured ontology information into natural language and applying a pre-trained natural language model to vectorize semantics. OSR2Vec converts directly linked ontology words into sentence sequences, which are then embedded to semantics representation using the ouBioBERT model and the TSDAE method. We evaluate our method on a protein semantic similarities task using the kgsim dataset. We demonstrate the advantages of OSR2Vec by constructing sentence sequence to attain more specific semantics and confirm that performance on the protein semantic similarities task is favorable when using a combination of ouBioBERT and TSDAE according to our method.
\end{abstract}

Conclusions: OSR2Vec is a method to represent ontology semantics using pre-training natural language model embedding sentences. With increasing the quality of representation, OSR2Vec provides an effective method in the ontology-based semantic task.

\section{Introduction}

In different biomedical databases, the same concept is likely to be named differently, and even the same term may express different meanings[1]. Due to the difference in expression, it is difficult to use the database word set as annotation resource for correct semantic queries, data integration, and knowledge mining[2]. As a way of conceptualizing domain knowledge, ontology provides a standardized formal definition of domain knowledge and is ideal for biological data annotation[3]. The unit of description in an ontology is the term, which is meaningfully related to one another and organized in a tight hierarchical framework[4]. And biomedical ontologies are widely used to provide semantic metadata in biological databases. For example, Gene Ontology(GO) annotates the Uniprot databases[5], and Human Phenotype 
Ontology (HPO) provides annotations for diseases in the Orphanet database[6], and Disease ontology (DO) annotates human genes in the context of disease[7].

The biological entities annotated by ontology terms can be compared, quantified at the semantic level. The ontology similarity measure is a special kind of semantic similarity measure[8], which provides a means to compare entities on aspects that would otherwise not be comparable. Peculiarly, semantic similarity evaluates the degree of association between two entities by their similarity in the annotation sense[9]. Semantic similarity calculation methods can be divided into two categories based on theoretical principles. Firstly, the calculation paradigm based on information content(IC) similarity employs the occurrence probability of terms in the domain corpus to enhance the ontology's classification knowledge. The previous method[10] and its variants[11, 12] calculate the most informative common ancestor (MICA) of two terms in ontology to reflect semantic similarity. Secondly, when the ontology is viewed as a hierarchical geometric structure, the similarity of terms can be estimated according to their relative distance in the ontology structure. And representatively, Wang et al[13] estimated the similarity based on the graph structure of GO. These methods have achieved strong results in semantic similarity analysis, whether based on the information content or geometric structure.

Recently, machine learning approaches have garnered increased attention in ontology semantic similarity analysis. Some researchers applied language models such as Word2vec[14], Onto2Vec[15], and OPA2Vec[16] to similarity analysis using ontology. And Onto2Vec and OPA2Vec are word2vec's variants. These models are used to generate feature vectors and apply them to semantic similarity analysis tasks like protein function prediction and gene-disease prediction, among others[17, 18]. These methods have in common that train the vector by taking into account several types of ontology information, including asserted and inferred logical axioms, datatype properties, and annotation axioms. However, word2vec cannot solve the problem of polysemous words because there is a one-to-one correspondence between words and vectors. Thus word-sensitive features is therefore no longer accurate as a semantic feature for annotating. An alternative approach is to substitute Word2Vec with Bidirectional Encoder Representation from Transformers(BERT), which generates representation for the word based on its context[19]. BERT has been applied in ontology-based analysis tasks as the research progresses. Duong et al[20] implement BERT to produce GO embeddings, and define three tasks to evaluate the GO embeddings. Wang et al[21] propose OntoSem, a method that leverages bert to produce ontology semantic representation in the biological domain, which is utilized for cross-ontology similarity comparison and protein-protein function prediction tasks.

Generally, these neural network language models convert ontology terms into computable vectors. However, ontology terms are usually sequences of several words, and the performance of the sentence-level embedding is insufficient by BERT[22]. At present, some sentence embedding techniques, InferSent[23], Universal Sentence Encoder (USE) [24], and SBERT[25] have been developed, but they rely heavily on labeled data to train models. In addition, ontology semantics information is loaded by both on terms selves and the relationship contained in the hierarchy, which implies that the long sequence is essential for more semantically expression. Kexin Wang et al[26] proposed a new approach, Transformer-based Sequential Denoising 
Auto-Encoder (TSDAE), which uses more semantically rich sentences as training predictions. TSDAE trains decoder by reconstructing the original sentences from sentence embedding, contributing to encoder to capture detailed semantic features.

In this paper, to contribute a gap that the previous method on the representation of ontology semantics is insufficient for ontology-based semantics application, we develop OSR2Vec, a method for ontology semantic representation. Specifically, to learn the best semantic characteristics tailored to an ontology, we first transform the hierarchical relationship between terms to semantic sentences, where terms in the ontology are directly related. With the pre-training model whose training on the extracted sentence corpus, we apply the TSDAE approach for domain adaptation, and then generate vector representations for ontology terms. Then we apply OSR2Vec to generate dense vector representation of ontology as annotations so as for semantic-based tasks. And we evaluate our method on the kgsim dataset[27], a publically available dataset, which provides a collection of benchmark datasets for researchers to use to evaluate the similarity measures of proteins with three different proxies.

\section{Methods}

We show a diagram of the method in this section. Figure 1 depicts a framework of OSR2Vec. The framework consists three main aspects: corpus construction, model specification and ontology embedding. Firstly, we construct corpus by extracting sentences derived from ontology terms and relationships that contain their hierarchical relationships. Then the corpus is fed into BERT model, which transforms word sequences into fixed-sized vectors. We used the TSDAE approach for further capturing semantic features from sentences by adding a certain type of noise to input sentences. After being trained, OSR2Vec encodes vector for the terms utilized for annotations or description in the ontology. Details of each aspect is described as follows.

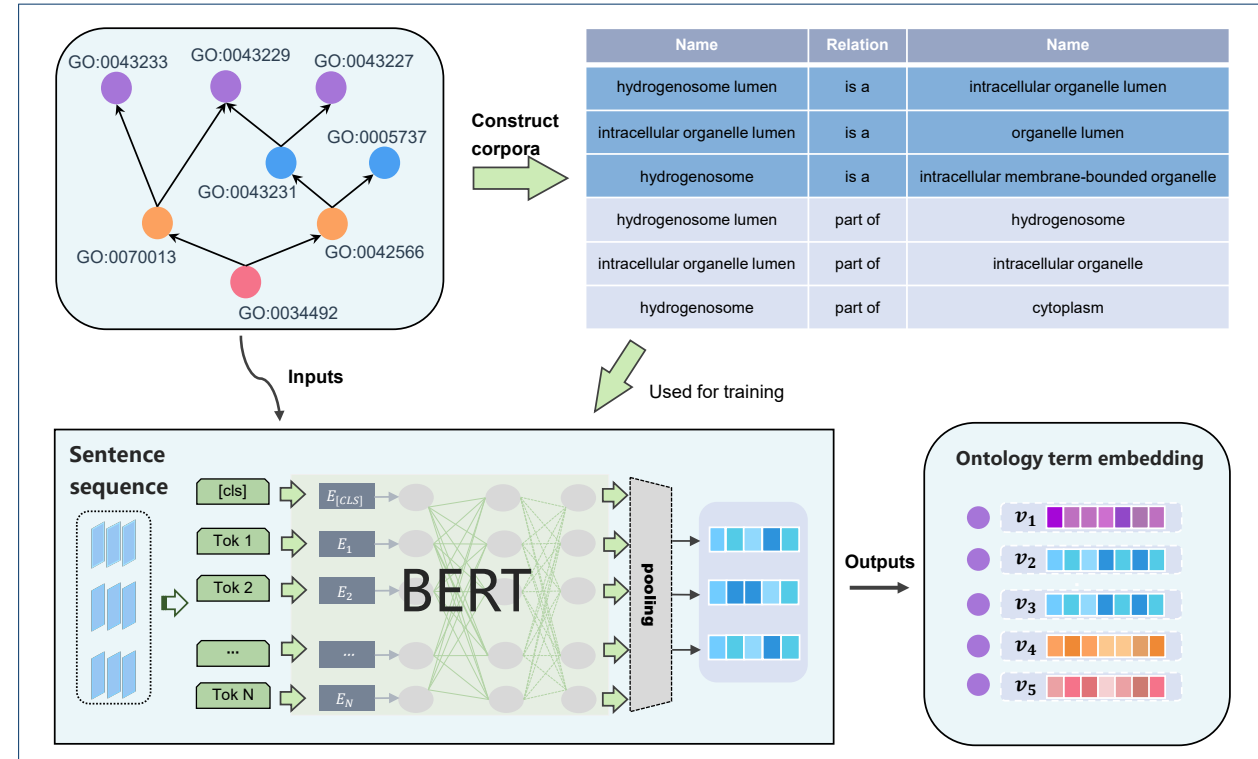

Figure 1 The processing pipeline of OSR2Vec. 


\section{Corpus construction}

The structure of the ontology could be regarded as a directed acyclic graph, with each term representing a node in the graph. There are many different types and numbers of relationships between nodes, all of which show unambiguous directivity. In general, those nodes that are directly related, i.e. neighbor nodes, are father-child relationships or inclusion relationships or others, which have a high level of semantic similarity. To transitive the semantic between ontology terms without damage, we build the corpus by the following procedures: To start with, we view the ontology as a graph consisting of connected nodes and edges. Then, these nodes are all traversed accordingly. During the traversal phase, all neighbor nodes and relational edges for each node are retrieved to construct sentence sequences. Namely, we extract the closely related concept pairings between the hierarchy of the ontology into sentences. And finally, we create 180,910 unsupervised sentences from GO that annotate the subsequently mentioned dataset, to fine-tune OSR2Vec.

\section{Model specification}

We apply BERT on the corpus to transform the sentences into their vector representations. BERT is a machine learning model whose embeddings style is contextualized, namely mutual information maximization[28]. BERT is optimized in two pre-training tasks proposed by the original paper that is Masked Language Model(MLM) and Next Sentence Prediction(NSP). We briefly describe the key idea of the two tasks by the form of inputs. The input sequence of BERT is first transformed into token sequence. And each token contains three types of embeddings: Token Embedding, Segment Embedding, and Positioning Embedding, as shown in Figure2. The input is partitioned into the following tokens [CLS] mi\#ch\#drial DNA meta\#\# process part of [SEP] \#toch\#drial ge\#\# maintenance [SEP]. The first token of every sequence is always a special token [CLS], which corresponding to the whole inputs for classification tasks. And the token $[\mathrm{SEP}]$ is utilized to differentiate the sentences together with positioning embedding. The masked form is just like the "\#" operator, which makes the model does not know whether the vocabulary at the corresponding input position is the correct vocabulary. Therefore, the MLM task makes the model rely more on contextual information to predict vocabulary. The task of NSP estimates whether the two paragraphs of text input to BERT are continuous text, which makes the model understand the relationship between two sentences. In this work, we opt for the ouBioBERT model[29], which is a language model based on the BERT-Base architecture and was pre-trained on PubMed abstracts from the PubMed baseline.

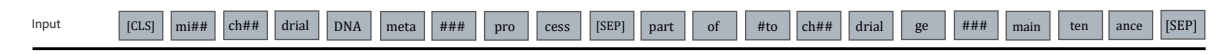

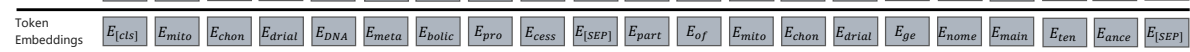

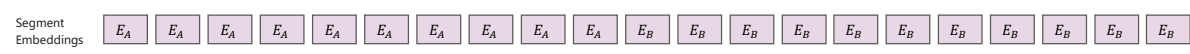

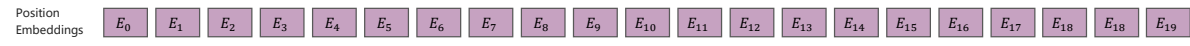

Figure 2 BERT input representation of sentence sequence.

As noted previously, we extract sentences with relatively higher semantic expression from the ontology's hierarchical structure and then embed the sentences. 
Generally, the average pooling strategy outperforms the [CLS] strategy over BERT context embeddings in unsupervised learning task, particularly semantic text similarity task[22]. And some researches have shown that the straightforward use of the BERT approach performs poorly comparing the similarity between two sentence embeddings [25, 26]. To offset the weakness, we employ the TSDAE approach based on BERT to produce semantic embedding. TSDAE is a learning method used for unsupervised sentence embedding based on pre-trained Transformers and sequential denoising autoencoder via an encoder-decoder architecture. The idea of TSDAE is similar to MLM that is the method for introducing noise, forcing the encoder to capture the complete meaning of the sentence. More specifically, TSDAE introduces noises by removing some input words randomly, which enforces the encoder to produce a semantical sentence representation. It has been proved that TSDAE is more suitable to start from pre-trained transformers, which can utilize the pretrained knowledge while avoiding overfitting problems[26]. In this work, we inherit the setup of TSDAE from the original paper and apply ouBioBERT to yield an encoded vector that describes the global information of the input text.

\section{Ontology embedding}

The fine-tuned model, trained on the sentence corpus extracted, is used as an encoder to semantically encode entities including ontology terms. The basic concept in ontology is a term, which contains primary elements such as unique numeric identifier, name, category, definition, and relationship. To obtain the loaded semantics of ontology, we need to conform to the structure of the ontology. For this purpose, we connect the term's name and definition to a long sequence as input text because they inherently contain a large proportion of semantical information. Then we input them into the fine-tuned model to generate embeddings. Taking one term with the GO id GO:0004690 as an example, we concatenate its name and definition to the long sequence, namely cyclic nucleotide-dependent protein kinase activity cNMP-dependent catalysis of the reaction: ADP + a phosphoprotein Equals ATP + a protein. After that, the model encodes the long sequence into a fixed-length sentence vector representing the semantic feature of the term.

\section{Experiment Settings}

Dataset

We evaluate our OSR2Vec ability to represent ontology semantics, by adopting the public kgsim dataset to carry out the proteins semantic similarity. The dataset contains tweenty protein benchmark datasets based on GO annotation to evaluate measures of proteins semantic similarity according the similarity proxies.

The protein benchmark dataset comprises proteins recognized by their UniProt Accession Numbers and each protein is annotated by GO terms. The dataset includes four species datasets: Drosophila Melanogaster(DM), Escherichia Coli(EC), Homo Sapiens(HS), and Saccharomyces Cerevisiae(SC), as well as their aggregated ALL dataset. According to the annotated GO term aspects, each dataset is separated into two types: One aspect and All aspects. The following are their definitions: 'One aspect': The proteins must have at least one annotation in each GO aspect, and in at least one aspect, there should be at least one leaf-class annotation. 'All 
aspects': The proteins must have at least one annotation in each GO aspect, and in each aspect, there should be at least one leaf-class annotation.

These datasets evaluate the similarity calculation method by the Pearson correlation coefficient of similarity proxy methods. The protein benchmark datasets similarity proxies are sequence similarity, molecular functional similarity, and proteinprotein interactions similarity. We briefly describe the relationship between these proxies and semantic similarity. First of all, sequence similarity refers to the the likelihood for sequence homology of two proteins. Though the relationship between semantic similarity and sequence similarity is not linear, the higher the sequence similarity, the more relevant the semantics[30,31]. It is important to note that it should not be utilized as the sole criterion, while sequence similarity can be used as a proxy indicator to measure semantic similarity. Secondly, the principle underlying molecular function similarity is that the more functional domains of two proteins share, the higher semantic similarity in the Molecular Function aspect in GO[32]. And thirdly, protein-protein interactions(PPIs) mean if two proteins appear at a certain location in a cell at the same time and participate in the same cellular activity. Therefore, they are likely to interact and share common GO terms in the Biological Process and Cellular Component aspects, which exhibit the correlation in PPIs with semantic similarity[33]. In the following, for convenience of explanation, we use the similarity proxy method to refer to the dataset, such as the molecular function datasets and the protein-protein interactions datasets. Due to the difference of One aspect and All aspects, each of the protein-protein interactions dataset and molecular function dataset has ten datasets, as shown in table1.

Table 1 Statistical information on the benchmark datasets.

\begin{tabular}{|c|c|c|c|c|c|}
\hline \multicolumn{2}{|c|}{ Species } & \multicolumn{2}{c|}{ One aspect } & \multicolumn{2}{c|}{ All aspects } \\
\cline { 3 - 6 } & & Proteins & Pairs & Proteins & Pairs \\
\hline \multirow{4}{*}{ PPI } & D. melanogaster & 455 & 364 & 287 & 200 \\
\cline { 2 - 6 } & E. coli & 371 & 734 & 263 & 420 \\
\cline { 2 - 6 } & H. sapiens & 7093 & 30826 & 6718 & 29672 \\
\cline { 2 - 6 } & S. cerevisae & 3776 & 27898 & 2888 & 16904 \\
\cline { 2 - 6 } & All & 11695 & 59822 & 10156 & 47196 \\
\hline \multirow{4}{*}{ MF } & D. melanogaster & 7470 & 31350 & 5300 & 17682 \\
\cline { 2 - 6 } & E. coli & 1231 & 3363 & 724 & 1332 \\
\cline { 2 - 6 } & H. sapiens & 13246 & 31350 & 11666 & 25527 \\
\cline { 2 - 6 } & S. cerevisae & 4782 & 38166 & 3660 & 29265 \\
\cline { 2 - 6 } & All & 26729 & 104229 & 21350 & 73806 \\
\hline
\end{tabular}

\section{Compare methods}

OSR2Vec applies TSDAE adding noises to the input of ouBioBERT to generate semantic embeddings. And TSDAE follows the recommendations of the original paper. The key of TSDAE is the use of deletion as input noise with a noise ratio of 0.6. We chose the average pooling strategy out of the three pooling strategies of cls, mean, and max because it is much better than the other two ways. To verify the effectiveness of our proposed method, we compare OSR2Vec against traditional similarity computation methods, namely BMAResnik, BMASeco, simGICResnik, simGICSeco, bma_lin method and bma_wang method. The first four approaches are separated into two groups: the BMA method and the simGIC method. According to different IC calculation methodologies, each category is subdivided into Resnik and Seco. More details of these methods can be found in Appendix. The bma_lin 
method and bma_wang method are the Lin's measure and Wang's measure combined with the BMA approach respectively. And the bma_lin method is modification of the information degree calculation of the two terms' most informative common ancestor[12]. The bma wang method is based on the graph structure, which relies on the relationship between the positions of two terms in the ontology graph and their ancestors[13].

\section{Result}

As shown in Figure 3, we can find that the best performance approach for these datasets is consistent between "One aspect" and "All aspect", indicating that there is usually the same one method constantly performs best on a specific dataset. And the simGIC-based approach performed poorly compared to the BMA and OSR2Vec. For EC1, EC3, DM1 and DM3 datasets, our proposed method leads to reach the performance by $0.72,0.70,0.80$ and 0.85 scores of Pearson correlation coefficients. For most of the datasets, OSR2Vec can consistently perform comparably with the methods based on BMA. The only exception is ALL dataset where the BMA Resnik method is the most efficient. We believe this is because the ALL dataset is compiled from all datasets and contains a high level of information concentration, reflecting the benefits of information content and the BMA strategy as the size of datasets grows.

\begin{tabular}{|c|c|c|c|c|c|c|c|c|}
\hline ALL1 - & 0.899 & 0.587 & 0.597 & 0.553 & 0.677 & 0.679 & 0.695 & \multirow{2}{*}{$\begin{array}{r}1.0 \\
-0.9\end{array}$} \\
\hline ALL3 - & 0.893 & 0.547 & 0.558 & 0.539 & 0.649 & 0.650 & 0.672 & \\
\hline DM1 - & 0.858 & 0.841 & 0.718 & 0.719 & 0.857 & 0.846 & 0.835 & \multirow{2}{*}{-0.8} \\
\hline DM3 - & 0.805 & 0.803 & 0.688 & 0.690 & 0.806 & 0.800 & 0.795 & \\
\hline $\mathrm{ECl}-$ & 0.700 & 0.710 & 0.609 & 0.624 & 0.722 & 0.711 & 0.708 & -0.7 \\
\hline EC3 - & 0.674 & 0.689 & 0.590 & 0.604 & 0.702 & 0.695 & 0.694 & \multirow{2}{*}{-0.6} \\
\hline HS1 - & 0.489 & 0.505 & 0.379 & 0.389 & 0.494 & 0.499 & 0.490 & \\
\hline HS3 - & 0.493 & 0.509 & 0.380 & 0.390 & 0.500 & 0.503 & 0.493 & -0.5 \\
\hline $\mathrm{SC} 1-$ & 0.646 & 0.633 & 0.562 & 0.563 & 0.642 & 0.650 & 0.638 & -0.4 \\
\hline SC3 - & 0.620 & 0.613 & 0.537 & 0.538 & 0.612 & 0.621 & 0.609 & \multirow[b]{2}{*}{-0.3} \\
\hline & BMA $R e^{S N^{\prime}}$ & ${ }_{B M A} S^{S^{C^{\circ}}}$ & GICResnik & $\mathrm{G}^{1} \mathrm{SS}^{\mathrm{C}^{\mathrm{O}}}$ & ospzec & pma-lin & bma wang & \\
\hline $\begin{array}{l}\text { Figure } 3 \\
\text { aspect" } \\
\text { The dep } \\
\text { the dark }\end{array}$ & $\begin{array}{l}\text { Pearso } \\
\text { type wh } \\
\text { th of the } \\
\text { er the b }\end{array}$ & $\begin{array}{l}\text { orrelation } \\
\text { SC3 indic } \\
\text { olor speci } \\
\text { the lowe }\end{array}$ & $\begin{array}{l}\text { coefficien } \\
\text { tes "All } \\
\text { cally indi } \\
\text { the simil }\end{array}$ & $\begin{array}{l}\text { of PPls } \\
\text { ects" ty } \\
\text { tes that } \\
\text { ty. }\end{array}$ & $\begin{array}{l}\text { res of } \mathrm{di} \\
\text { and the } \\
\text { darker }\end{array}$ & $\begin{array}{l}\text { rent met } \\
\text { hers are } \\
\text { red, the }\end{array}$ & $\begin{array}{l}\text { lods. SC1 } \\
\text { lassified ir } \\
\text { higher the }\end{array}$ & $\begin{array}{l}\text { tes "One } \\
\text { same way } \\
\text { arity, and }\end{array}$ \\
\hline
\end{tabular}

Figure 4 shows the results with sequence similarity. It can be found that the correlation between semantic similarity and sequence similarity compared with protein-protein interactions is loose on all measures. In contrast to the PPIs, the magic-based approach performed better in sequence similarity. On SC, HS, and DM datasets, the method based on simGIC yields the highest scores of $0.35,0.56$, and 


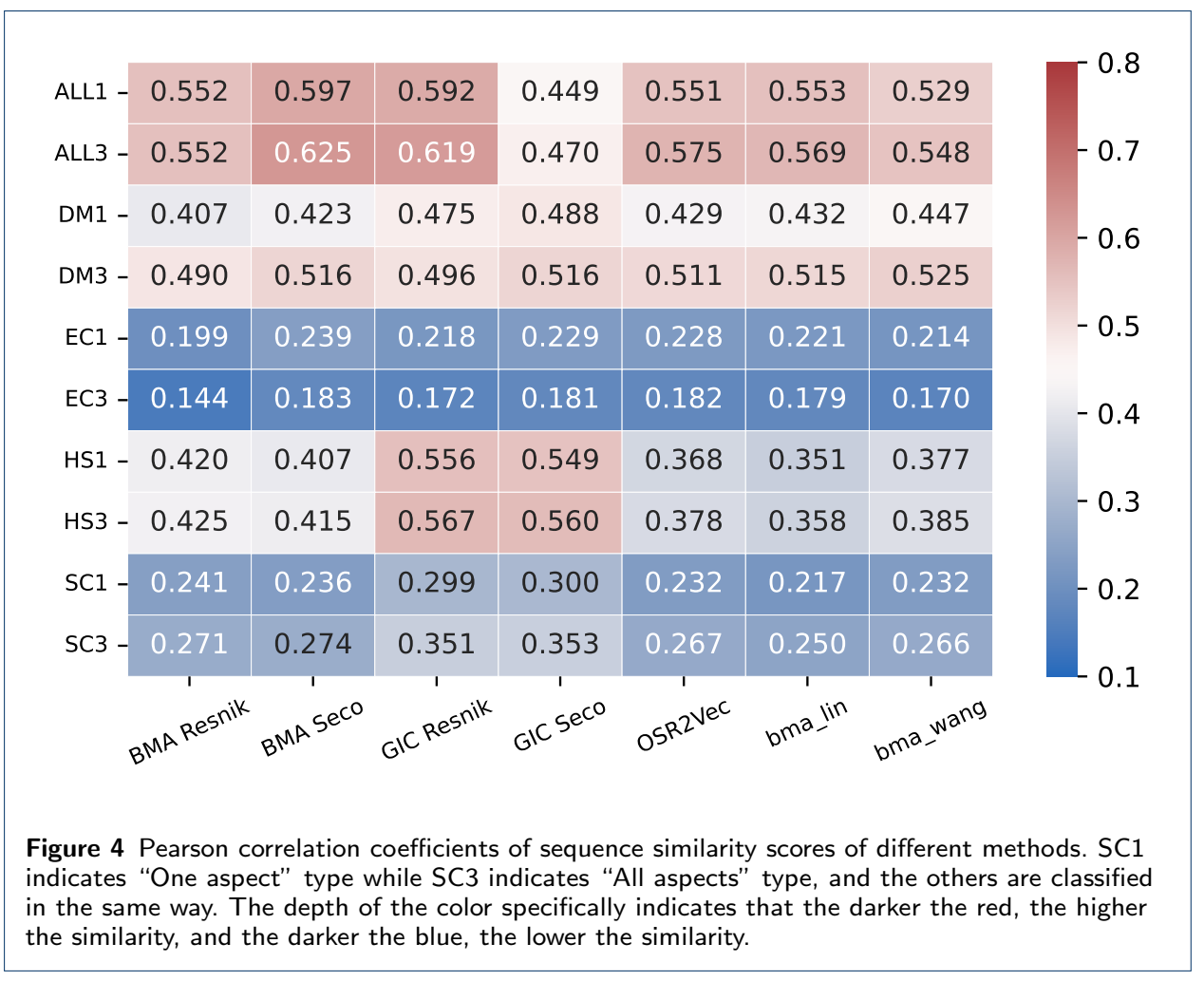

0.51 respectively. Depressedly, we find OSR2Vec presents a relatively low score. The main reason is that our method works in obtaining the semantic characteristics contained in ontology terms, which reflect sparsely, or almost no about the sequence information. We plot the distribution of an average number of annotation genes for these datasets in Figure 5. We see that the EC and SC datasets have a smaller number of annotation genes corresponding to the lower scores in figure 5 . And this suggests that the insufficient annotation genes are the possible reason that leads to lower sequence similarity.

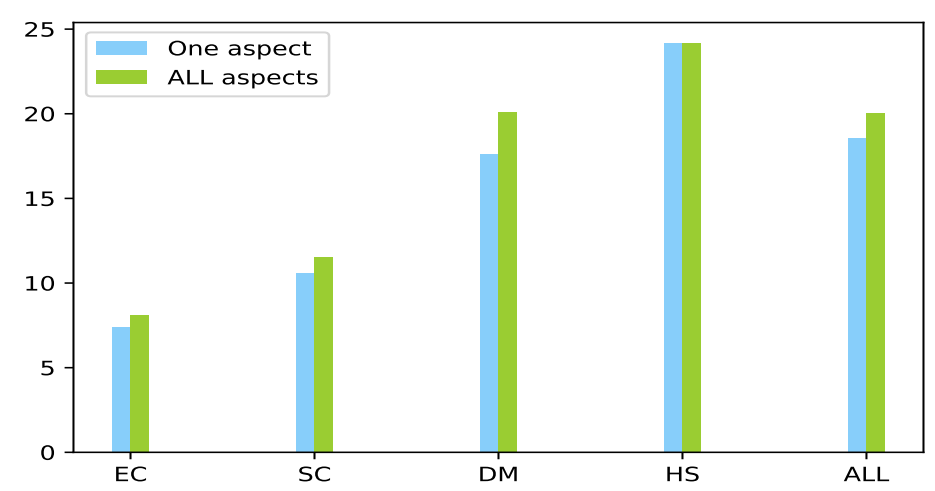

Figure 5 Distribution of an average number of annotation genes for EC, SC, DM, HS and ALL datasets on PPIs. 


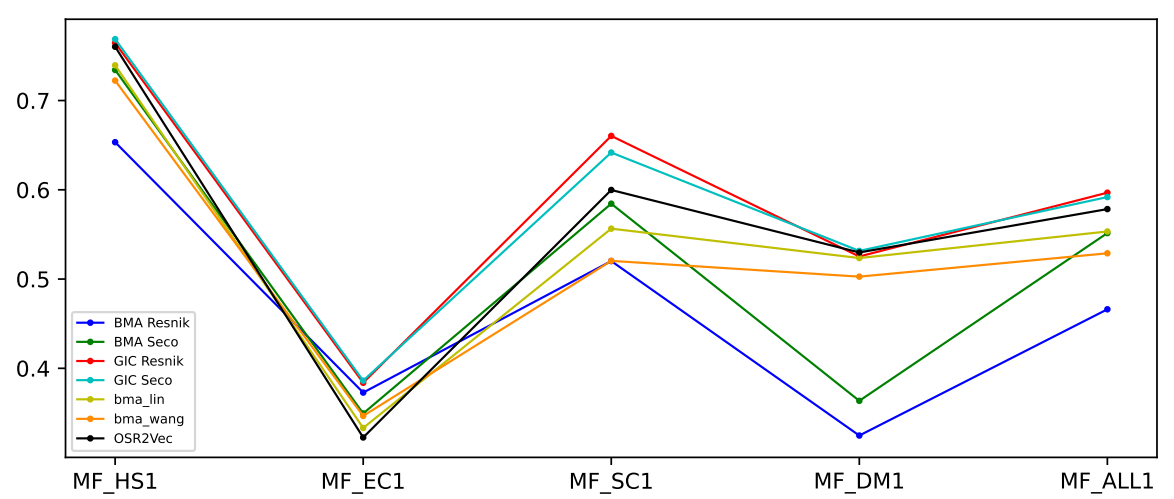

(a) The distribution of sequence similarity on "One aspect".

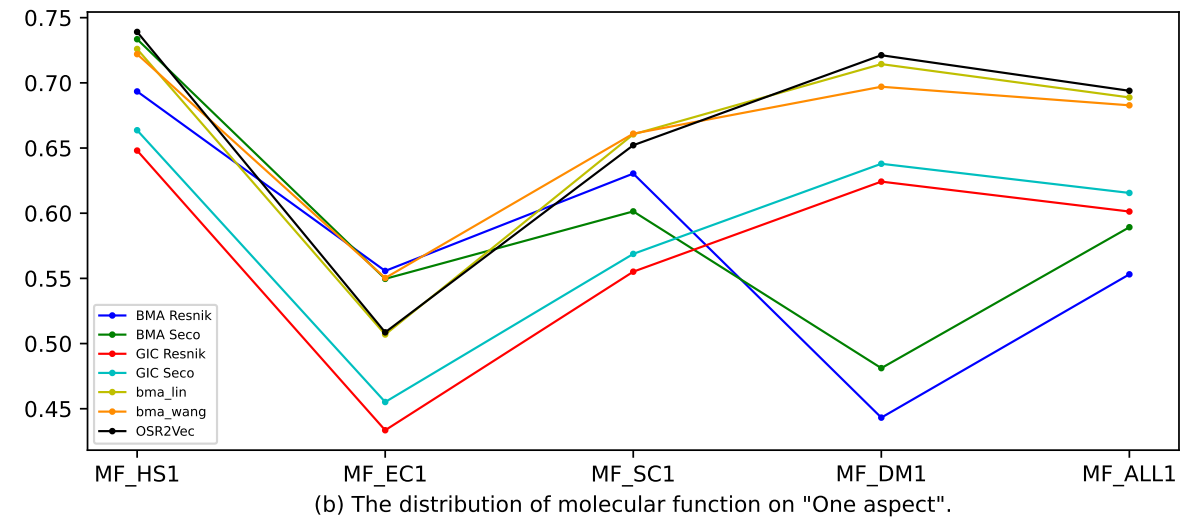

Figure 6 The diagram distribution of Pearson correlation coefficient of sequence similarity and molecular function on One aspect. The solid line represents the Pearson correlation coefficient score of sequence similarity and molecular function distributed on different datasets. Different colored lines reflect different methods.

Figures 6 demonstrate the Pearson correlation coefficient scores of sequence similarity and molecular function from the four species datasets in terms of "One aspect". Within sequence similarity, we observed that each method's performance varied greatly on five types of datasets almost in Figure 6 (a). MF_HS1 dataset appears to show a better correlation with sequence than others in all methods, while MF_EC1 dataset shows dramatically lower correlation. And the performance of sequence similarity and molecular function on MF_SC1, MF_DM1, and MF_ALL1 show significant differences among measures. As shown in Figure 6 (b), OSR2Vec gives the best performance of molecular function on the HS1, DM1, and ALL1 datasets except for EC1 and SC1 datasets. And on SC1, bma_lin and bma_wang methods work slightly better than OSR2Vec. There, too few annotated genes lead to insufficient representation of semantic information. As shown in Figure 7, the average number of annotated genes on the EC dataset is less than ten, which suggests the main reason leading to the relatively lower performance of OSR2Vec. According to the comparison results, our method can improve the performance and achieve $0.739,0.721$, and 0.693 on the HS1, DM1, and ALL1 datasets respectively. This result indicates that our method offers an advantage over others within the molecular functions similarity of proteins. 


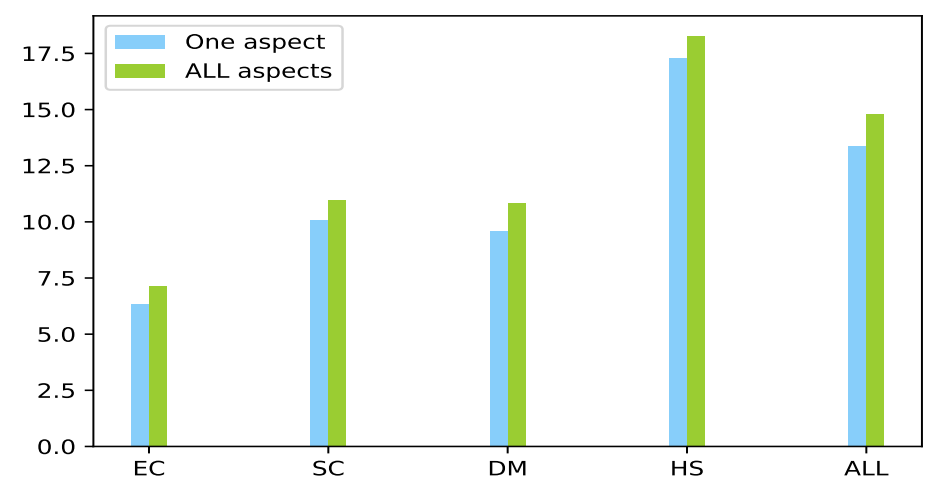

Figure 7 Distribution of an average number of annotation genes for EC, SC, DM, HS and ALL datasets on MF.

Figures 8 demonstrate the Pearson correlation coefficient scores of sequence similarity and molecular function from the four species datasets in terms of "All aspects". The trend of the line graphs of all approaches in One aspect and All aspects are almost identical. We observe that the methods based on simGIC work well on sequence similarity continuously while performing poorly on molecular function. It also presents that the result of OSR2Vec is relatively balanced when compared to other methods. Except for EC3, as shown in figure 8 (b), our method continues to outperform the others on the HS3, SC3, DM3, and ALL3 datasets. Limited by the small number of average annotated genes in the EC dataset, OSR2Vec is at a disadvantage compared with other methods. On the majority of datasets, we confirm that OSR2Vec can improve protein molecular function prediction when compared to other approaches. Overall, the results show that our method could effectively ontology embeddings for the semantic similarity task.

\section{Discussion}

Among possible ontology semantic strategies, our method concentrates on sentence sequence which can be focused on by language models to be aware of similarities. Generally, the highest expectation is to attain the exhaustive semantic information and then thoroughly absorb it by highly interpretable representation form for semantic similarity tasks or other subsequent applications. Sentences can express more meaningful semantics multi-level in-depth than discrete words and phrases, and the ontology interior knowledge introduced by effective sentences can be helpful for machine learning approaches to work well on mentioned tasks. Thus constructing sentences concerning related terms to capture ontology semantics, OSR2Vec performs well on the similarity calculation task of the biomedical dataset. In addition, we address that OSR2Vec represents ontology semantics with high attention in the "computable" dimension. To stay sensitive and loyal in biological and biomedical, OSR2Vec applies the combination of the TSDAE method with the ouBioBERT language model, which is pre-trained on the biomedical domain, a variant of BERT. The utilization of a domain-specific model enhances representation capacity. Furthermore, the TSDAE method helps capture the semantics of sentences formed of 


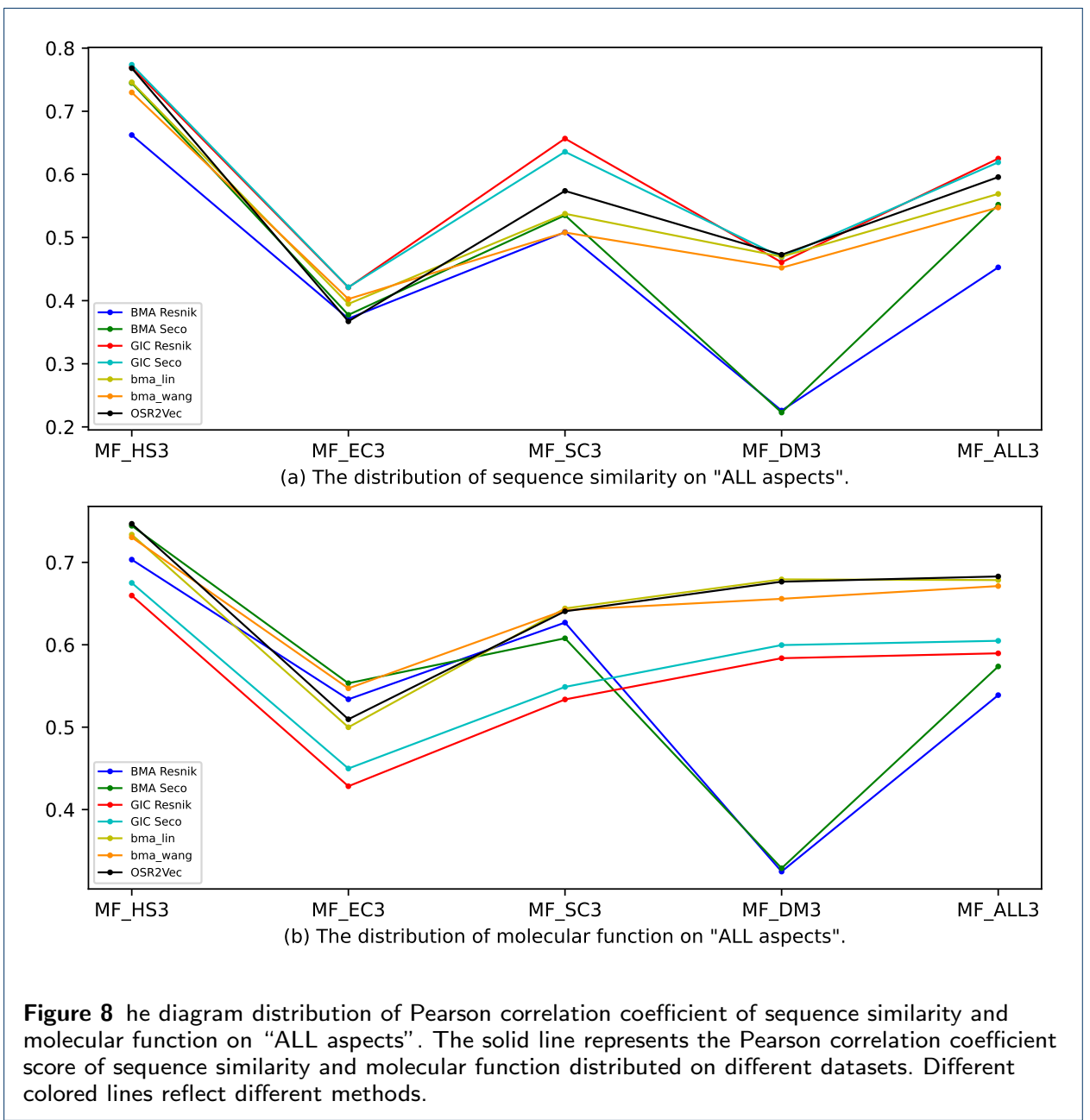

terms and relations more precisely in embedding semantics contained in the ontology.

And according to the experiment, we observe that OSR2Vec shows a favorable performance in the molecular function. OSR2Vec works well in molecular function analysis because proteins are annotated by GO terms whose molecular function aspect, one of three sub-ontologies, is ideal for protein molecular function analysis. Having relatively poor performance in sequence similarity, OSR2Vec does not capture the sequence characteristics contained in proteins by annotation meaning from ontology terms for semantic representation.

OSR2Vec provides an approach in ontology semantics representation, nevertheless, the comprehensiveness of our method should be viewed due to the following limitation, which we intend to address in future work. OSR2Vec exclusively extracts sentences with semantic relations from the ontology for the most directly linked, leaving other possible associations in the hierarchical structure unaccounted for, perhaps resulting in partial semantic information loss. In the future, we would aim at representing multi-level in-depth ontology information, such as mining potential semantics through undirect links between concepts and training using negative samples. Another interesting approach could be to combine the external knowledge 
bases such as knowledge graphs, which will capture the semantic characteristics of ontology terms more comprehensively and authoritatively, bringing more surprises to ontology-based similarity analysis tasks.

\section{Conclusion}

In this paper, we offer a method for calculating ontology term semantic similarity. We create a natural language corpus with good readability and interpretability by extracting closely related terms from the ontology. And we introduce TSDAE to fine-tune the ouBioBERT model to build semantically sentence embedding that represent the semantic features of ontology. In the experiments, we evaluate our OSR2Vec on three proxies of the protein semantic similarities: sequence similarity, PPIs prediction, and molecular function. The experimental results show that our method outperforms previous methods for PPIs prediction, and molecular function tasks, suggesting that OSR2Vec has an effective contribution to increase the semantic representation of ontology.

Availability of data and materials

The developed method is freely available on GitHub at https://github.com/3waterya/OSR2Vec.

Authors' contributions

Huiting Sun design computer programs, perform the experiments, and write the manuscript; Junjie Wang and Jie $\mathrm{Hu}$ conceive the ideas or evolution of overarching research goals and development of methodology, and review the manuscript; Wei Feng and Xinyi Cao verify, whether as a part of the activity or separate, of the overall

reproducibility of experiments; Yun Liu oversight the leadership responsibility for the research activity planning and execution and provide the financial support.

Corresponding author Correspondence to Yun Liu.

Abbreviations

GO: Gene Ontology

HPO: Human Phenotype Ontology

DO: Disease ontology

DM: Drosophila Melanogaster

EC: Escherichia Coli

HS: Homo Sapiens

SC: Saccharomyces Cerevisiae

ALL: the collection of all datasets

Acknowledgements

We would like to thank the equipment support provided by Institute of Medical Informatics and Management, Nanjing Medical University.

Funding

This work has been supported by funding from the the industry prospecting and common key technology key projects of Jiangsu Province Science and Technology Department (Grant No. BE2020721), the Special guidance funds for service industry of Jiangsu Province Development and Reform Commission (Grant No.[2019]1089), the big data industry development pilot demonstration project of Ministry of Industry and Information Technology of China (Grant No. [2019]243, [2020]84), the Industrial and Information Industry Transformation and Upgrading Guiding Fund of Jiangsu Economy and Information Technology Commission(Grant No. [2018]0419), the Research Project of Jiangsu Province Sciences(Grant No. 2019-2020ZZWKT15), the found of Jiangsu Engineering Research Center of Jiangsu Province Development and Reform Commission (Grant No.[2020]1460), the found of Jiangsu Digital Future Integration Innovation Center (Grant No.[2018]498).

Competing interests

Not applicable

Ethics approval and consent to participate

Not applicable

Consent for publication

Not applicable

Author details

${ }^{1}$ Department of Medical Informatics, School of Biomedical Engineering and Informatics, Nanjing Medical University, Nanjing, China. ${ }^{2}$ Institute of Medical Informatics and Management, Nanjing Medical University, No.300 Guang

Zhou Road, Nanjing, Jiangsu, 210029, China. 
References

1. Noy, N.F.: Semantic integration: A survey of ontology-based approaches. SIGMOD Rec. 33(4), $65-70$ (2004). doi:10.1145/1041410.1041421

2. Zheng, S., Wang, F., Lu, J.: Enabling ontology based semantic queries in biomedical database systems. International Journal of Semantic Computing 08(01), 67-83 (2014). doi:10.1142/S1793351X14500032. https://doi.org/10.1142/S1793351X14500032

3. Kaiya, H., Saeki, M.: Using domain ontology as domain knowledge for requirements elicitation. In: 14th IEEE International Requirements Engineering Conference (RE'06), pp. 189-198 (2006). doi:10.1109/RE.2006.72

4. OGREN, P.V., COHEN, K.B., ACQUAAH-MENSAH, G.K., EBERLEIN, J., HUNTER, L.: THE COMPOSITIONAL STRUCTURE OF GENE ONTOLOGY TERMS, pp. 214-225. doi:10.1142/9789812704856 021 .https://www.worldscientific.com/doi/abs/10.1142/9789812704856 021

5. Consortium, T.U.: UniProt: a worldwide hub of protein knowledge. Nucleic Acids Research 47(D1), 506-515 (2018). doi:10.1093/nar/gky1049.

https://academic.oup.com/nar/article-pdf/47/D1/D506/27437297/gky1049.pdf

6. Köhler, S., Carmody, L., Vasilevsky, N., Jacobsen, J.O., Danis, D., Gourdine, J.-P., Gargano, M., Harris, N.L., Matentzoglu, N., McMurry, J.A., Osumi-Sutherland, D., Cipriani, V., Balhoff, J.P., Conlin, T., Blau, H., Baynam, G., Palmer, R., Gratian, D., Dawkins, H., Segal, M., Jansen, A.C., Muaz, A., Chang, W.H. Bergerson, J., Laulederkind, S.J., Yüksel, Z., Beltran, S., Freeman, A.F., Sergouniotis, P.I., Durkin, D., Storm, A.L., Hanauer, M., Brudno, M., Bello, S.M., Sincan, M., Rageth, K., Wheeler, M.T., Oegema, R., Lourghi, H., Della Rocca, M.G., Thompson, R., Castellanos, F., Priest, J., Cunningham-Rundles, C., Hegde, A., Lovering, R.C., Hajek, C., Olry, A., Notarangelo, L., Similuk, M., Zhang, X.A., Gómez-Andrés, D., Lochmüller, H., Dollfus, H., Rosenzweig, S., Marwaha, S., Rath, A., Sullivan, K., Smith, C., Milner, J.D., Leroux, D., Boerkoel, C.F., Klion, A., Carter, M.C., Groza, T., Smedley, D., Haendel, M.A., Mungall, C., Robinson, P.N.: Expansion of the Human Phenotype Ontology (HPO) knowledge base and resources. Nucleic Acids Research 47(D1), 1018-1027 (2018). doi:10.1093/nar/gky1105. https://academic.oup.com/nar/article-pdf/47/D1/D1018/27437514/gky1105.pdf

7. Schriml, L.M., Mitraka, E., Munro, J., Tauber, B., Schor, M., Nickle, L., Felix, V., Jeng, L., Bearer, C., Lichenstein, R., Bisordi, K., Campion, N., Hyman, B., Kurland, D., Oates, C.P., Kibbey, S., Sreekumar, P., Le, C., Giglio, M., Greene, C.: Human Disease Ontology 2018 update: classification, content and workflow expansion. Nucleic Acids Research 47(D1), 955-962 (2018). doi:10.1093/nar/gky1032. https://academic.oup.com/nar/article-pdf/47/D1/D955/27437186/gky1032.pdf

8. Shvaiko, P., Euzenat, J., Jiménez-Ruiz, E., Cheatham, M., Hassanzadeh, O.: Ontology matching: Om-2018: Proceedings of the iswc workshop. In: OM 2018-13th ISWC Workshop on Ontology Matching, pp. 1-227 (2018). No commercial editor.

9. Pesquita, C., Faria, D., Falcão, A.O., Lord, P., Couto, F.M.: Semantic similarity in biomedical ontologies. PLOS Computational Biology 5(7), 1-12 (2009). doi:10.1371/journal.pcbi.1000443

10. Resnik, P.: Using information content to evaluate semantic similarity in a taxonomy. ArXiv abs/cmp-lg/9511007 (1995)

11. Jiang, J.J., Conrath, D.W.: Semantic similarity based on corpus statistics and lexical taxonomy. In: ROCLING/IJCLCLP (1997)

12. Lin, D., et al.: An information-theoretic definition of similarity. In: Icml, vol. 98, pp. 296-304 (1998)

13. Wang, J.Z., Du, Z., Payattakool, R., Yu, P.S., Chen, C.-F.: A new method to measure the semantic similarity of GO terms. Bioinformatics 23(10), 1274-1281 (2007). doi:10.1093/bioinformatics/btm087. https://academic.oup.com/bioinformatics/article-pdf/23/10/1274/497100/btm087.pdf

14. Jatnika, D., Bijaksana, M.A., Suryani, A.A.: Word2vec model analysis for semantic similarities in english words. Procedia Computer Science 157, 160-167 (2019)

15. Smaili, F.Z., Gao, X., Hoehndorf, R.: Onto2vec: joint vector-based representation of biological entities and their ontology-based annotations. Bioinformatics 34(13), 52-60 (2018)

16. Smaili, F.Z., Gao, X., Hoehndorf, R.: Opa2vec: combining formal and informal content of biomedical ontologies to improve similarity-based prediction. Bioinformatics 35(12), 2133-2140 (2019)

17. Alshahrani, M., Khan, M.A., Maddouri, O., Kinjo, A.R., Queralt-Rosinach, N., Hoehndorf, R.: Neuro-symbolic representation learning on biological knowledge graphs. Bioinformatics 33(17), 2723-2730 (2017)

18. Alshahrani, M., Hoehndorf, R.: Semantic disease gene embeddings (smudge): phenotype-based disease gene prioritization without phenotypes. Bioinformatics 34(17), 901-907 (2018)

19. Devlin, J., Chang, M.-W., Lee, K., Toutanova, K.: Bert: Pre-training of deep bidirectional transformers for language understanding. arXiv preprint arXiv:1810.04805 (2018)

20. Duong, D., Uppunda, A., Gai, L., Ju, C., Zhang, J., Chen, M., Eskin, E., Li, J.J., Chang, K.-W.: Evaluating representations for gene ontology terms. biorxiv, 765644 (2020)

21. Zhao, L., Wang, J., Cheng, L., Wang, C.: Ontosem: an ontology semantic representation methodology for biomedical domain. In: 2020 IEEE International Conference on Bioinformatics and Biomedicine (BIBM), pp. 523-527 (2020). IEEE

22. Li, B., Zhou, H., He, J., Wang, M., Yang, Y., Li, L.: On the sentence embeddings from pre-trained language models. arXiv preprint arXiv:2011.05864 (2020)

23. Conneau, A., Kiela, D., Schwenk, H., Barrault, L., Bordes, A.: Supervised learning of universal sentence representations from natural language inference data. arXiv preprint arXiv:1705.02364 (2017)

24. Cer, D., Yang, Y., Kong, S.-y., Hua, N., Limtiaco, N., John, R.S., Constant, N., Guajardo-Cespedes, M., Yuan, S., Tar, C., et al.: Universal sentence encoder for english. In: Proceedings of the 2018 Conference on Empirical Methods in Natural Language Processing: System Demonstrations, pp. 169-174 (2018)

25. Reimers, N., Gurevych, I.: Sentence-bert: Sentence embeddings using siamese bert-networks. arXiv preprint arXiv:1908.10084 (2019)

26. Wang, K., Reimers, N., Gurevych, I.: Tsdae: Using transformer-based sequential denoising auto-encoder for 
unsupervised sentence embedding learning. arXiv preprint arXiv:2104.06979 (2021)

27. Cardoso, C., Sousa, R.T., Köhler, S., Pesquita, C.: A collection of benchmark data sets for knowledge graph-based similarity in the biomedical domain. Database 2020 (2020)

28. Kong, L., d'Autume, C.d.M., Ling, W., Yu, L., Dai, Z., Yogatama, D.: A mutual information maximization perspective of language representation learning. arXiv preprint arXiv:1910.08350 (2019)

29. Wada, S., Takeda, T., Manabe, S., Konishi, S., Kamohara, J., Matsumura, Y.: Pre-training technique to localize medical bert and enhance biomedical bert. arXiv preprint arXiv:2005.07202 (2020)

30. Lord, P.W., Stevens, R.D., Brass, A., Goble, C.A.: Investigating semantic similarity measures across the gene ontology: the relationship between sequence and annotation. Bioinformatics 19(10), 1275-1283 (2003)

31. Ikram, N., Qadir, M.A., Afzal, M.T.: Investigating correlation between protein sequence similarity and semantic similarity using gene ontology annotations. IEEE/ACM Transactions on Computational Biology and Bioinformatics 15(3), 905-912 (2017)

32. El-Gebali, S., Mistry, J., Bateman, A., Eddy, S.R., Luciani, A., Potter, S.C., Qureshi, M., Richardson, L.J., Salazar, G.A., Smart, A., et al.: The pfam protein families database in 2019. Nucleic acids research 47(D1), 427-432 (2019)

33. Sousa, R.T., Silva, S., Pesquita, C.: Evolving knowledge graph similarity for supervised learning in complex biomedical domains. BMC bioinformatics 21(1), 1-19 (2020) 


\section{Supplementary Files}

This is a list of supplementary files associated with this preprint. Click to download.

- Supplementarylnfo.docx 